\section{Military Technical College Kobry El-kobbah, Cairo, Egypt}

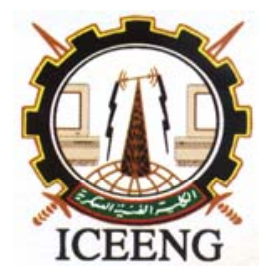

\title{
PERSONAL IDENTIFICATION SYSTEM BASED ON HUMAN IRIS RECOGNITION
}

\section{$5^{\text {th }}$ International Conference on Electrical Engineering ICEENG 2006}

\author{
Osama* Fathy Hegazy
}

\begin{abstract}
The biometric person identification technique based on the pattern of the human iris is a very costly top secure application. This paper describes a personal identification system based on the human iris imaging through providing a set of algorithms that describes image acquisition, image segmentation, feature extraction and pattern forming. For image acquisition, we present an image enhancement algorithm in order to get more accurate image feature results. In addition, we propose a boundary localization algorithm, which used to find the pupil boundary. A new iris recognition method that analyzes local variations of the iris is used to construct a Feature Selection Vector (FSV) that can be used to extract features of any iris image size. Extensive experimental results using Pearson's correlation coefficient to verify one's identity on CASIA iris images database shows that the proposed system is effective and encouraging.
\end{abstract}

Keywords: Iris Recognition, Biometric Identification, Image Segmentation, Pattern Recognition, Pearson's Correlation Coefficient.

\section{INTRODUCTION}

With an increasing emphasis on security, automated personal identification based on biometrics has been receiving extensive attention over the past decade. Biometrics [10],[25] aims to accurately identify each individual using various physiological or behavioral characteristics, such as fingerprints, face, iris, retina, gait, palm-prints and hand geometry etc. Recently, iris recognition is becoming an active topic in biometrics due to its high reliability for personal identification [5],[17],[24]. The human iris, an annular part between the pupil and the white sclera has an extraordinary structure and provides many interlacing minute characteristics such as freckles, coronas, stripes, furrows, crypts and so on. These visible characteristics, generally called the texture of the iris, are unique to each subject. The uniqueness of the iris pattern is the direct result of the individual differences that exist in the development of the anatomical structures in the body. Some research work [4],[8],[11],[22$24]$ has also stated that the iris is essentially stable over a person's life. Furthermore, since the iris is an internal organ as well as externally visible, iris-based personal identification systems can be noninvasive to their users [4-7],[11],[22-24], which is of great importance for practical applications. All these desirable properties make iris recognition a particularly promising solution to security.

* Phd. Computer Science Department, Cairo Higher Institute for Computers, Information and Management, Cairo Academy, Golf Region, Cairo, Egypt. 
This paper divided into two main parts. The first one describes the preprocessing techniques through the following sections: Section 2 reports a history of previous related works and methods. Image acquisition introduced in Section 3. Image segmentation and feature extraction mentioned in Section 4 and Section 5 respectively. Pattern forming showed Section 6. The second part uses data generated by the first part of the paper for pattern identification through the following sections: Section 7 includes iris matching via Pearson's Correlation Coefficient. Experimental results and conclusions illustrated in Section 8 and Section 9 respectively. Fig. 1 illustrates an overview of the proposed system.

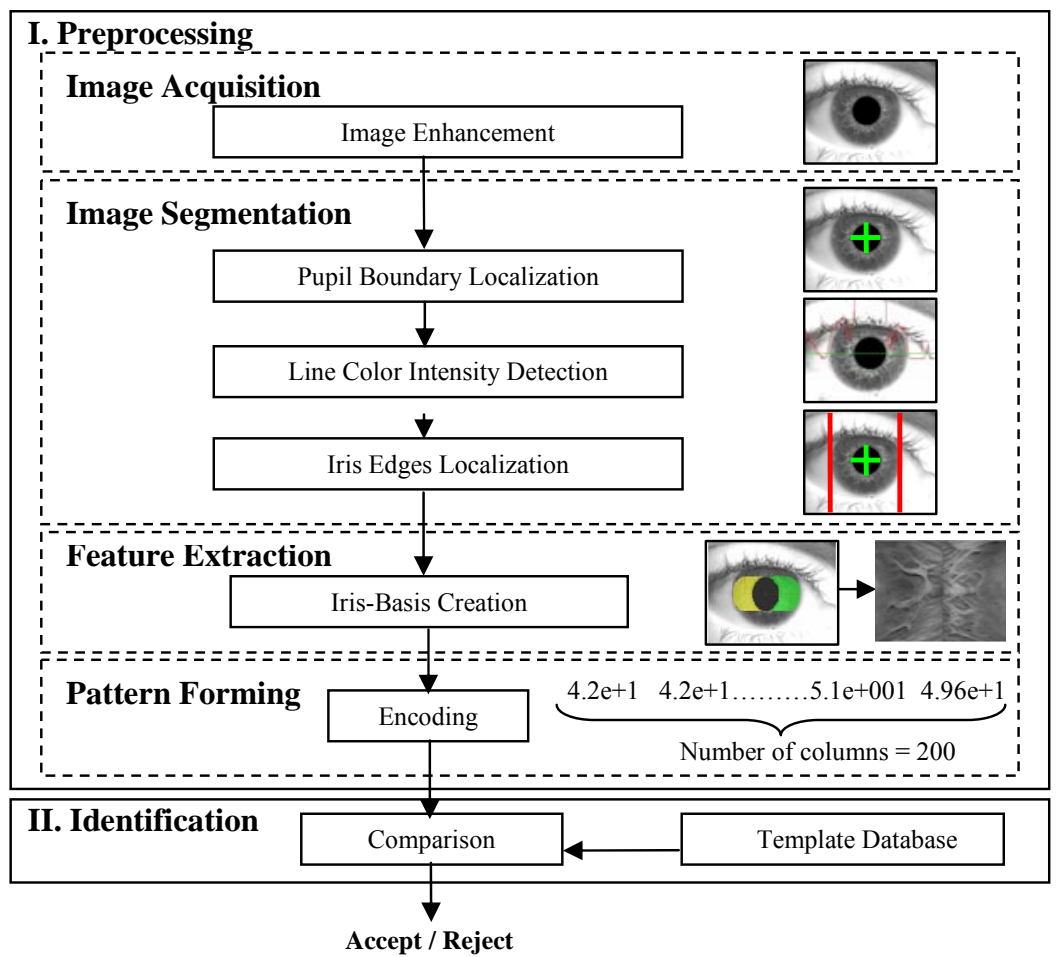

Fig. 1 Iris Recognition System Phases.

\section{RELATED WORK}

Flom and Safir first proposed the concept of automated iris recognition in 1987 [8]. Since then, some researchers worked on iris representation and matching and have achieved great progress [2],[4-7],[11],[13-15],[18],[20-24],[26]. In the literature, there are four main approaches to iris representation: first method is called phase-based methods, where Daugman [5]-[7] made use of multiscale Gabor filters to demodulate texture phase structure information of the iris. Filtering an iris image with a family of filters resulted in 1024 complex-valued phasors, which denote the phase structure of the iris at different scales. Each phasor was quantized to one of the four quadrants in the complex plane. The resulting 2048component iris code was used to describe an iris. The difference between a pair of iris codes was measured by their Hamming distance. The second method is called Zero-crossing representation, where Boles and Boashash [2] calculated a zero-crossing representation of one-dimensional wavelet transform at various resolution levels of a concentric circle on an iris image to characterize the texture of the iris. Iris matching was based on two dissimilarity functions. Boles et al. in [1] attempted to use different similarity measures for matching, such as Euclidean distance and Hamming distance. The third method is called Texture analysis, where Wildes et al. [22] represented the iris texture with a Laplacian pyramid constructed with four different resolution levels and used the normalized correlation to determine whether 
the input image and the model image are from the same class. In [26], Zhu et al. attempts to iris recognition developed the texture analysis-based methods. Ma et al. in [15] attempt the global texture features of the iris were extracted by means of well-known Gabor filters at different scales and orientations. Based on the experimental results and analysis obtained in [14], Ma et al. in [11] constructs a bank of spatial filters, whose kernels are suitable for iris recognition, to represent the local texture features of the iris and thus achieved much better results. Park et al. [18] used a directional filter bank to decompose an iris image into eight directional sub-band outputs and extracted the normalized directional energy as features. Iris matching was performed by computing Euclidean distance between the input and the template feature vectors. The last method is called Intensity variation analysis, where Bae et al. [1] projected the iris signals onto a bank of basis vectors derived by independent component analysis and quantized the resulting projection coefficients as features. Ma et al. in [14-15] develops local intensity variations of the iris as features. In addition, he uses GaussianHermite polynomial functions as transform kernels belong to a class of orthogonal moments. This means that they produce minimal information redundancy.

However, the question of which approach is most suitable for extracting iris features has never been answered.

\section{PART I - PREPROCESSING}

This part describes the preprocessing techniques used to code the irisbasis in a form, through a set of steps, which used for person's identification.

\section{IMAGE ACQUISITION}

One of the major challenges of automated iris recognition system is to capture a high quality image of the iris. This step is one of the most important and deciding factors for obtaining a good result. A good and clear image eliminates the process of noise removal and helps in avoiding errors in calculations. The following points should be taken into consideration:

- The images of the iris should be acquired with sufficient resolution and sharpness to support recognitions. It is important to have good contrast in the interior iris pattern without resorting to a level of illumination that annoys the operator.

- The images should be well framed (i.e. centered).

- The noises in the acquired images should be eliminated as much as possible.

It has been noticed by testing various iris images that some images produce undesired results, that is; the code used to find the center of the pupil produces heterogeneous and thereby rejected output. There are many reasons why the output is inaccurate:

- The camera flash maybe reflected on the pupil resulting a big white spot inside the pupil which may lead to inefficiency in calculating the pupil center.

- The high degree of eyelashes may overlap with the pupil leading inaccuracy in locating the pupil.

- The illumination of the image may lead to inaccuracy in locating the pupil, but applying a linear filter contrast can solve this problem.

- The noise in the image also leads to inaccuracy in locating the image; smoothing or blurring the image can solve this problem.

Therefore, to solve the last two problems, we apply the following enhancement algorithm:

\section{Image Enhancement Algorithm:}

1.Read a gray-scale image.

2.Adjust color intensity (contrast stretching technique) on the image as follows: 
- Calculate the histogram of the image and determines the adjustment limits automatically.

- Use a constant linear contrast filter (1.4 factors).

3.Apply Blurring on the image as follows:

- Average the pixels in an $\mathrm{n} \times \mathrm{n}$ size area.

- Convolute a Gaussian filter with an image.

The result of the enhancement algorithm is appeared in Fig. 2.

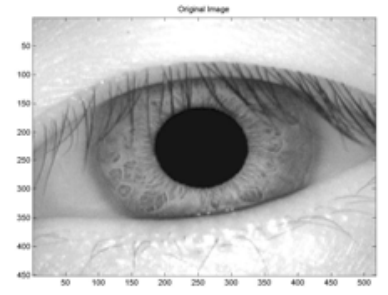

(a)

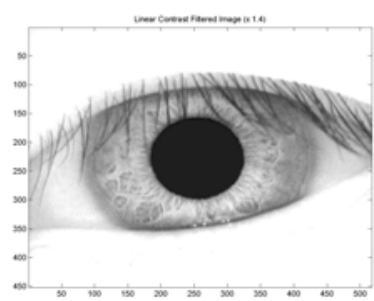

(b)

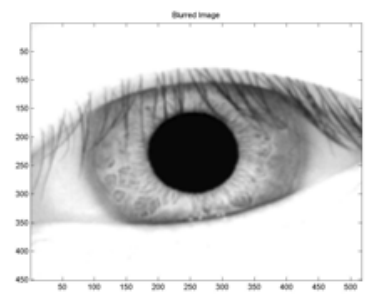

(c)

Fig. 2: (a) Original Eye image (b) Eye image after applying filter contrast

(c) Eye image after applying blurring

\section{IMAGE SEGMENTATION}

The main objective in this process is to remove the non-useful information, mainly the pupil segment and the part outside the iris (sclera, eyelids, skin). This process requires isolating the actual iris region in a digital eye image. The eyelids and eyelashes normally occlude the upper and lower parts of the iris region. In addition, secular reflections can occur within the iris region corrupting the iris pattern. A technique is required to isolate and exclude these artifacts as well as locating the circular iris region. The success of segmentation depends on the imaging quality of eye images. There are a large number of iris recognition methodologies that present almost optimal results, but only for well-segmented images [9].

\subsection{Pupil Boundary Localization}

Before performing iris feature extraction, the boundaries of the iris should be located. In other words, we are supposed to detect the part of the image that extends from inside the limbos to the outside of the pupil. Assuming that the iris region is already found in the face, and the image is windowed in the eye region (iris, sclera, eyelids), the area that constitutes the iris is very distinguishable because it has a concentration of pixels all of them with very low-level intensity (black or almost black). To find the pupil, we first need to apply a linear threshold on the image,

$$
g(x)=\left\{\begin{array}{l}
f(x)>70: 1 \text { (black) } \\
f(x) \leq 70: 0 \text { (white) }
\end{array}\right.
$$

Where $\boldsymbol{f}$ is the original image and $\boldsymbol{g}$ is the thresholded image. Pixels with intensity smaller than the empirical value of 70 (in a 0 to 256 scale) are dark pixels, therefore converted to 1 (black). Pixels greater than or equal to 70 are assigned to 0 (white).

Next, we apply Freeman's chain code to find regions of 8-connected pixels that are assigned with value equal 1 . It is possible to know that eyelashes also satisfy the threshold condition, but have a much smaller area than the pupil area. Using this knowledge, we can cycle through all regions and apply the following condition:

\section{for each region $R$ :}

$$
\text { if } \operatorname{AREA}(R)<2500 \text { : set all pixels of } R \text { to } 0
$$


Where: $\mathrm{R}$ is the number of pixels

The result of the thresholding process is appeared in Fig. 3.

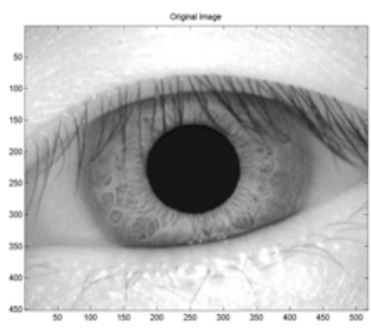

(a)

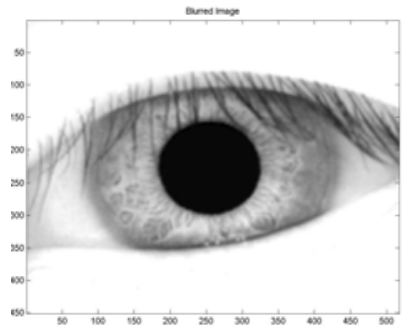

(b)

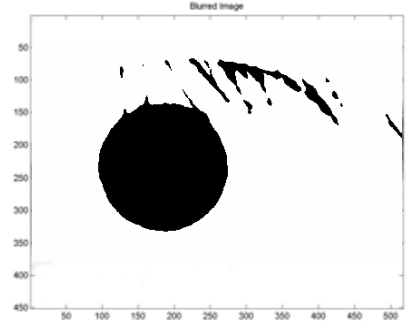

(c)

Fig. 3 (a) Original image. (b) Image after applying contrast and blurring (c) Image after threshold

Finally, we apply one last time the chain code algorithm in order to retrieve the only region in the image (hopefully the pupil). From this region, it is trivial to obtain its central moments [3]. Finding the edges of the pupil involves the creation of two imaginary orthogonal lines passing through the centroid of the region. The boundaries of the binarized pupil are defined by the first pixel with intensity zero, from the center to the extremities. Locating pupil boundaries are illustrated in the following algorithm and results is appeared in Fig. 4.

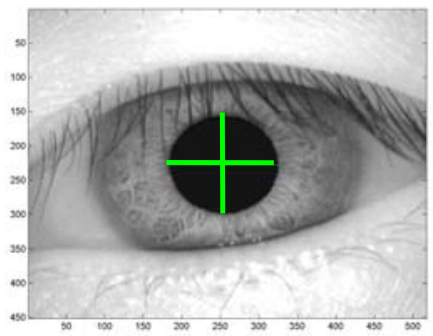

Fig. 4 Illustration of the horizontal and vertical radii passing through the center of the pupil

\section{Pupil Boundary Localization Algorithm:}

1.Apply linear threshold on the image, where all the pixels' color intensity with values smaller than 70 (in a 0 to 256 scale) are converted to 0 (white), while the values greater than 70 are converted to 1 (black).

2.Convert all the white regions to black and vice versa, get the number of all the white regions.

3.Convert the region of area less than 2500 (number of pixels) to black.

4.Repeat step 3 until all regions with area less than 2500 turn to black.

5.Get the coordinates of the center of the remaining white region of area greater than 2500 .

6.Get the horizontal and vertical lines that pass thought the center of the pupil.

7.Get the left and right edges of the pupil.

8.Get the upper and lower edges of the pupil.

\subsection{Line Color Intensity Detection:}

The color intensity of pixels of the imaginary line that crosses the whole image passing through the pupil center can be obtained by analyzing its signal from the center of the image towards the border. The imaginary line is divided into other two lines, a left line that starts from the first pixel in the imaginary line and ends at the left edge of the pupil, and a right line that starts from the right edge of the pupil and ends at the last pixel in the imaginary line as shown in Fig. 5. 


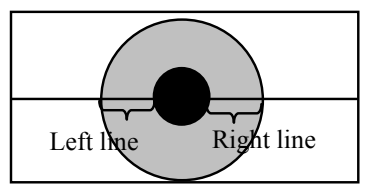

Figure 5 Illustration for the iris image showing the left and the right lines that pass through the pupil center

The intensities of both two lines should be detected to find the sudden change in intensity because the color of the iris is much darker than the color of the sclera. It is possible that some pixels inside the iris disk are very bright, causing a sudden rise in intensity. That could mislead the algorithm to detect that iris edge at that point. To prevent that from happening, the intensity average of small windows would be determined to detect the sudden rises of intensity. This process is cleared in the next algorithm, and Fig. 6 illustrates the results of that algorithm.

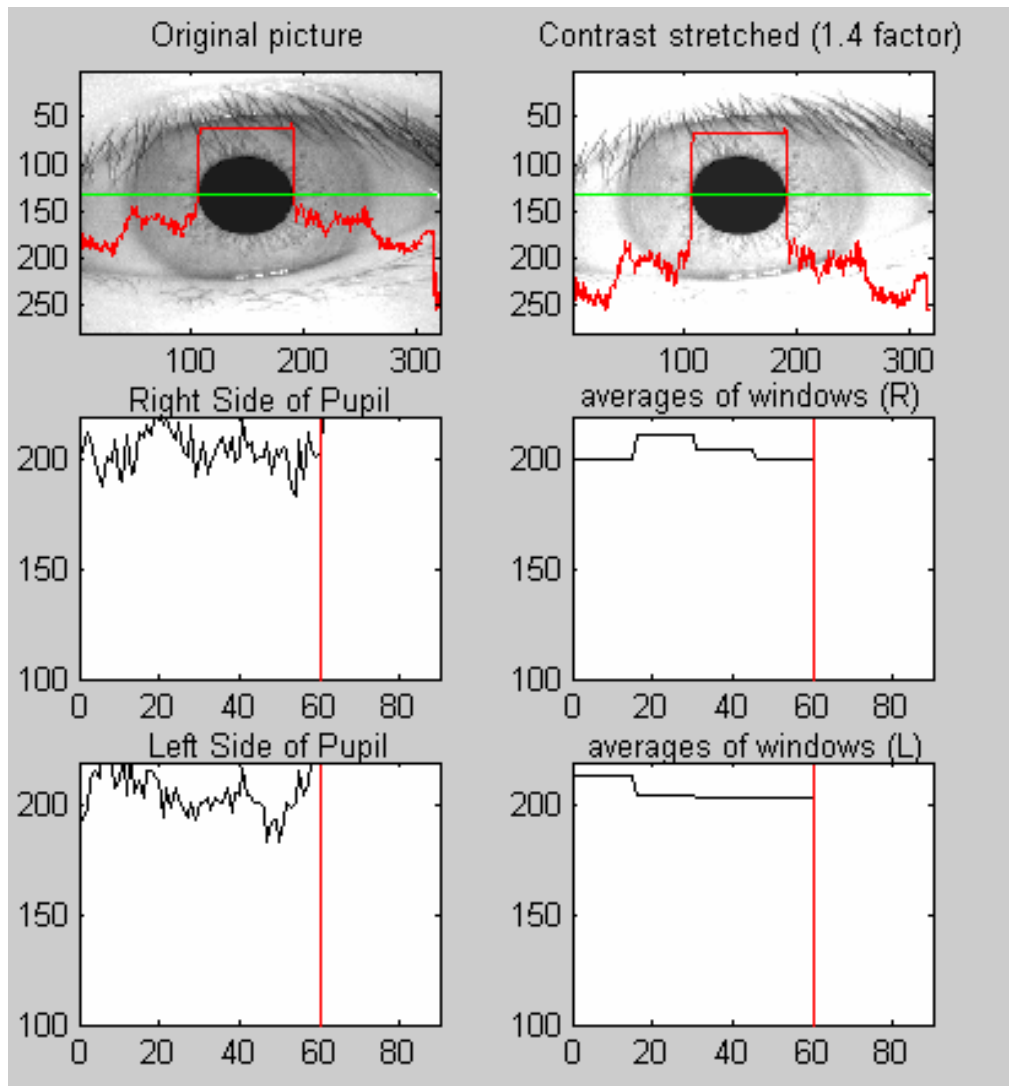

Fig. 6 Illustration for the real iris image showing the left and right edges of the iris, the intensities of both the left and the right lines and their averages of windows

\subsection{Iris Edges Localization}

The next process towards iris segmentation is finding the contour of the iris. Our method takes in consideration that areas of the iris at the right and left of the pupil are the ones that most often present visible to data extraction. The areas above and bellow the pupil also carry unique information, but it is very common that they are totally or partially occluded by 
eyelash or eyelid. The strategy adopted for iris detection uses the information from last section to trace a horizontal imaginary line that crosses the whole image passing through the center of the pupil. Starting from the edges of the pupil, we analyze the signal composed by pixel intensity from the center of the image towards the border and try do detect abrupt increases of intensity level. Although the edge between the iris disk and the sclera is most of the times smooth, it is known that it always have greater intensity than iris pixels. It is possible that some pixels inside the iris disk are very bright, causing a sudden rise in intensity, which could mislead the algorithm. To prevent that from happening, we take the intensity average of small windows and then detect when the sudden rises occur from these intervals. This process is presented in the next algorithm, and Fig. 7 shows the results of that algorithm.

\section{Iris Edges Localization Algorithm:}

1.Read a gray-scale image and adjust its contrast. Retrieve its pupil center, horizontal line that passes through it, and finally the radii values from Pupil Boundary Localization algorithm.

2.Add two margins to the left and right edges of the pupil on the horizontal line, and divide this line to left and right lines that start before and after both margins respectively.

3.Use Line Color Intensity algorithm to get and compute the position of both left and right iris edges where a sudden change in pixel intensity occurs.

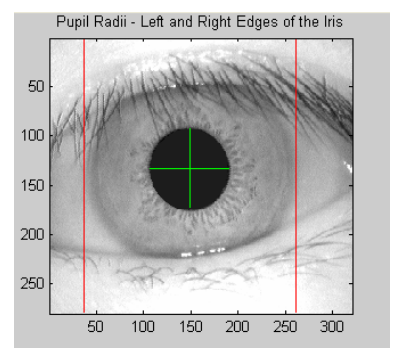

Fig. 7 Illustration of the horizontal and vertical radii passing through the center of the pupil.

\section{FEATURE EXTRACTION}

So far, we have performed the segmentation of the iris, first by finding the pupil and then finding the outer edge of the iris at the line that crosses the center of the pupil. The main reason for the prior segmentation is two folded. The first reason is to isolate only information that distinguishes individuals, namely the iris patterns. The second one is the attempt to reduce the size of pattern vector. For instance, the CASIA Iris Database provides images that are $320 \times 280$ pixels. If we concatenate all rows of the image into only one vector, the dimension of the problem would be to classify a vector with 89,600 elements. This dimension is too high for computing, and even though we had such capacity, satisfactory results is not guaranteed. So, Irisbasis is our first attempt to reduce the dimensionality of the problem while focusing only on parts of the scene that effectively identify the individual. In addition, we restrict the mapping of the iris to areas known to have less influence of eyelashes and eyelids, the sides of the iris. Assuming that intra-class rotation of iris is practically void, we propose an approach to extract pixels of either side of the pupil and forming one reduced image of the iris. This process is illustrated in the following algorithm, and the results are shown in Fig. 8.

\section{Irisbasis Creation Algorithm:}

1.Apply Iris Edges Localization algorithm to calculate the pupil diameter.

2.Extract pixels of either side of the pupil by setting the number of rows to transfer from top to bottom of the pupil $=200$, get the space between these lines.

3.Get the upper edge of the pupil and calculate the width of both left and right lines. 
4.Divide both left and right lines to 100 sections and calculate their start and ends by using Pythagoras theorem.

5.Get the color intensity of the pixels under both left and right sections.

6.Repeat Step 5 until all the pixels under the 100 sections in the left and right sides are obtained.

7.Repeat Step 4 through 6 until all the intensities of the 200 lines of both left and right lines are obtained.

8.Merge the left and right sides into one final matrix.

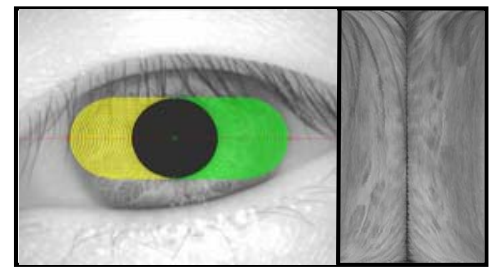

(a)

(b)

Figure 8 (a) Illustration of the iris image with pupil center line and the iris edge points in red. The vertical pupil markers and pupil center point in green. Yellow lines form the left half of the Irisbasis matrix, while the green lines form the other half. (b) The extracted Irisbasis image

\section{PATTERNS FORMING}

Dimension reduction was already achieved in the previous sections by simply isolating the area of the image that contains the characteristic information about an individual. Considering the fact that a large percentage of these pixels contain redundant information, we can utilize mathematical and statistical methods to reduce the dimensionality of the problem. This problem is also known as Feature Selection, from a set of $\boldsymbol{n}$ features, select a subset of $\boldsymbol{m}$ features that leads to minimum classification error. Reduction of irisbasis can be done by forming a pattern with 1 row and $\boldsymbol{m}$ columns. Obtain each row's color intensity values of irisbasis, and then calculate the sum of the obtained row's color intensity values. This process is illustrated in the following algorithm, where sample of 1 -D vector $(200 \times 1)$ irisbasis values is shown in Fig. 9.
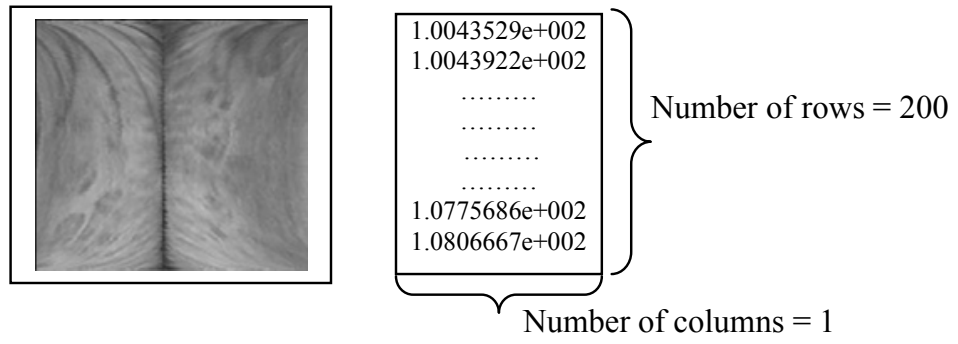

Fig. 9 Illustration of sample values of 1D irisbasis image values.

\section{Encoding Algorithm:}

1.Apply the Irisbasis Creation Algorithm to create irisbasis.

2.Read the color intensity values of irisbasis image and convert it to a double precision array.

3.Calculate the sum of the color intensity values of first row.

4.Repeat Step 2 for all rows, forming 1-D Feature Selection Vector (FSV) $(200 \times 1)$.

5.Append each irisbasis with its obtained FSV.

\section{PART II - IDENTIFICATION}


In the next section, we introduce a person identification method from the irisbasis image that pre-processed in "PART I" of this paper. By feature extraction, an iris image represented as a double precision feature vector. To reduce the computational cost and improve the identification accuracy, matching based on Pearson's product-moment correlation coefficient between input feature vector and feature vectors in database is used.

\section{MATCHING}

Pearson's product-moment correlation coefficient, $\mathbf{r}$, or simply the sample correlation coefficient, is a measure of extent to which two samples are linearly related. In addition, it can be used to calculate the similarity between two samples by using the formula:

$$
\mathrm{S}=(\mathbf{r}+1) / 2
$$

In general, sample $\mathbf{X}_{\mathrm{k}}$ has $\mathrm{n}$ measurements, or features, it can be written as $\mathbf{X}_{\mathrm{k}}=\left\{\mathrm{x}_{1 \mathrm{k}}, \mathrm{X}_{2 \mathrm{k}}\right.$, $\left.\ldots, \mathrm{x}_{\mathrm{nk}}\right\}$. There are many formulas that can be used to calculate $\mathrm{r}$, and the most detailed formula is given below:

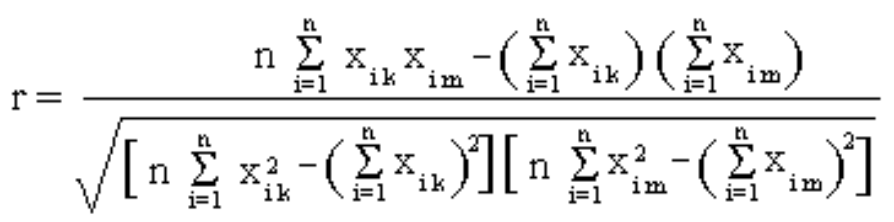

For any given two iris images; follow the pre-processing processes mentioned before, and form a FSV for each image. Then compute the similarity $\mathrm{S}$ between the two images by using (3), if $\mathbf{S}$ is greater than or equals to 0.93 , then the two images are matched, otherwise the two images did not. This process is illustrated in the following algorithm. Fig. 10 and Fig. 11 illustrates matching and non-matching between two irisbasis respectively.

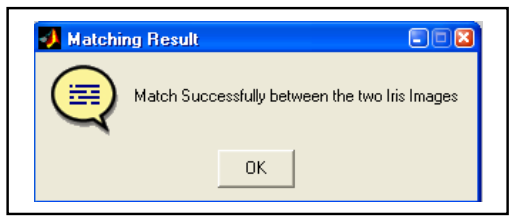

Fig. 10 Illustration of two matched irisbasis.
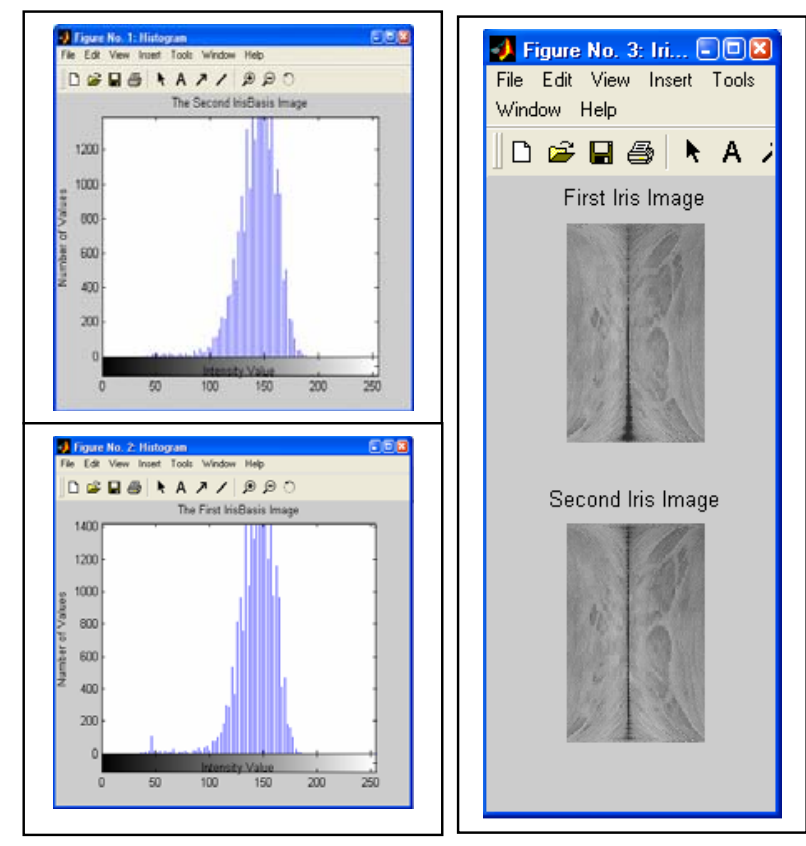


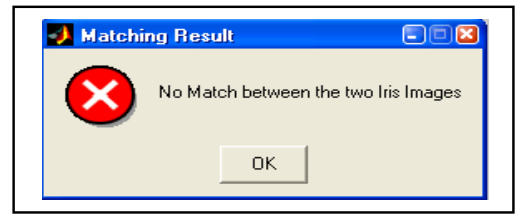

Fig. 11 Illustration of two nonmatched irisbasis.
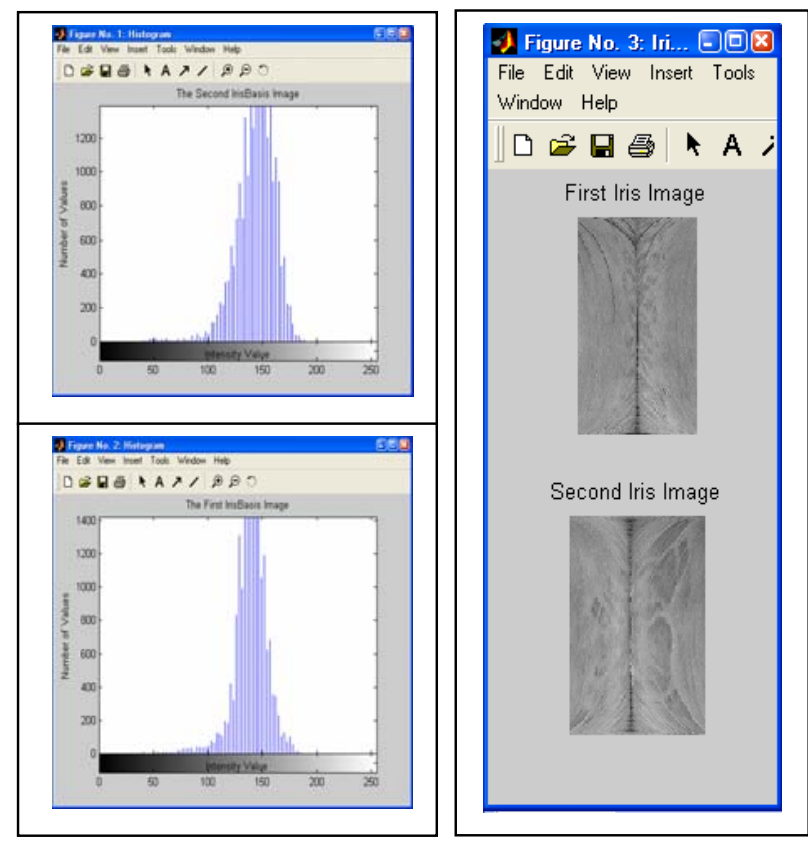

\section{Comparison between two Irisbasis Images Algorithm:}

1.Consider the system contains $\boldsymbol{k}$ images, each one appended with its Feature Selection Vector $(\boldsymbol{F S} \boldsymbol{V}-\boldsymbol{i}), \mathrm{i}=1,2, \ldots . ., \mathrm{k}$.

2.Read the irisbasis image $\boldsymbol{x}$ that required to be matched, and find its $\boldsymbol{F S} \boldsymbol{V}-\boldsymbol{x}$.

3. Compute the similarity $\boldsymbol{S}$ between $\boldsymbol{F} \boldsymbol{S} \boldsymbol{V}-\boldsymbol{x}$ and $\boldsymbol{F} \boldsymbol{S} \boldsymbol{V}-\boldsymbol{i}$ by using (3), i $=1,2, \ldots \ldots$, k, then

a- If $\mathrm{S}<0.93$, then no matching between image $\mathrm{x}$ and $\mathrm{i}=1,2, \ldots, \mathrm{k}$

b- If $\mathrm{S} \geq 0.93$ for only one image (image- $\boldsymbol{j}$ ), then matching exists between image- $\boldsymbol{x}$ and image- $j$.

c- If $S \geq 0.93$ for more than one image, then the highest $\boldsymbol{S}$ refers to image- $\boldsymbol{j}$ means matching exists between image- $\boldsymbol{x}$ and image- $\boldsymbol{j}$.

\section{EXPERIMENTS AND RESULTS}

To evaluate the performance of the proposed algorithms, we tested them on CASIA Iris Database [14]. The database includes 2255 iris images from 306 different eyes (hence, 306 different classes) of 213 subjects. The images are acquired during different sessions and the time interval between two collections is at least one month, which provides a challenge to our method. We conducted a comparative study of on CASIA Iris Database.

The experiments were completed in two modes: verification (one-to-one matching) and identification (one-to-many matching). In verification mode, the Receiver Operating Characteristic (ROC) curve and equal error rate (EER) are used to evaluate the performance of the proposed method. The ROC curve is a False Match Rate (FMR) versus False NonMatch Rate (FNMR) curve [14],[16-17], which measures the accuracy of matching process and shows the overall performance of a system. The FMR is the probability of accepting an imposter as an authorized subject and the FNMR is the probability of an authorized subject being incorrectly rejected. Points on this curve denote all possible system operating states in 
different tradeoffs. The EER is the point where the FMR and the FNMR are equal in value. The smaller the EER is, the better the algorithm.

The verification results are shown in Fig. 12 and Table 1. Fig. 12 is the ROC curve of the proposed method on the CASIA Iris Database. Points on this curve denote all possible system operating states in different tradeoffs. To exhibit the possible operating states more clearly, the horizontal axis of Fig. 12 is spread out using the logarithmic scale. The EER is only 1.59 $\%$. These results are quite encouraging and indicate the high performance of the proposed method. Table 1 lists three typical operating states of the proposed method. The experimental results demonstrate that the proposed iris representation is effective and local variations can well represent the differences between irises.

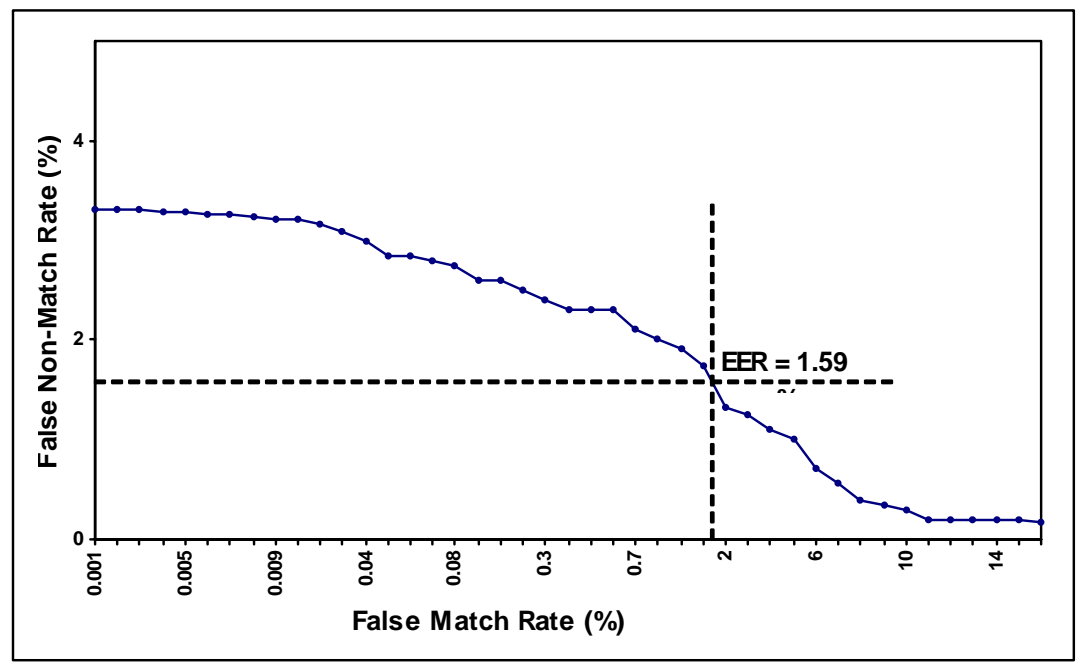

Fig. 12 The ROC curve of verification results

Table 1 The typical operating states of the proposed method

\begin{tabular}{|c|c|}
\hline False Match Rate (\%) & False Non-Match Rate (\%) \\
\hline 0.001 & 3.34 \\
\hline 0.01 & 1.39 \\
\hline 0.1 & 1.21 \\
\hline
\end{tabular}

In identification mode, the algorithm is measured by Correct Recognition Rate (CRR), the ratio of the number of samples being correctly classified to the total number of test samples. From all 2255 Iris images, this algorithm was able to correctly locate the center and radius of 2169 exemplars $(96.18 \%$ ) with a very low rate of failure (only 86 exemplars). Analyzing the images where the algorithm failed, we found out that most of them come from irises with high degree of eyelash overlapping and eyelids covering part of the iris. Eyelash is not a new problem in iris recognition research, Kong and Zhang [12] presented a study on how to detect eyelashes within an iris picture. The above experiments were performed in Matlab 6.5 on a $1700-\mathrm{MHz}$ PC with $512 \mathrm{M}$ RAM.

A comparison between our method and some previous works is shown in Table 2. From the results, we can find that Daugman's and Ma's methods has the best performance, our method is better than methods described in [2], [19] and [24] respectively.

Finally, different images for different individuals have been loaded; the experiments shows the authorized and unauthorized individuals; who belongs to the current database as shown in 
Fig. 13 and Fig. 14. In addition, the system can announce if the captured image should be rejected if the illumination affected the pupil size (narrowed it), and request recapturing the image as shown in Fig. 15.

Table 2 Comparison between our method and some previous works

\begin{tabular}{|c|c|c|}
\hline Methods & Correct Recognition Rate (\%) & Equal Error Rate (\%) \\
\hline Poursaberi [19] & 95.71 & - \\
\hline Boles [2] & 92.64 & 8.13 \\
\hline Daugman [7] & 100 & 0.08 \\
\hline Ma [14] & 100 & 0.07 \\
\hline Proposed & 96.18 & 1.59 \\
\hline Ma [15] & 99.19 & 0.57 \\
\hline Wildes [24] & - & 1.76 \\
\hline
\end{tabular}

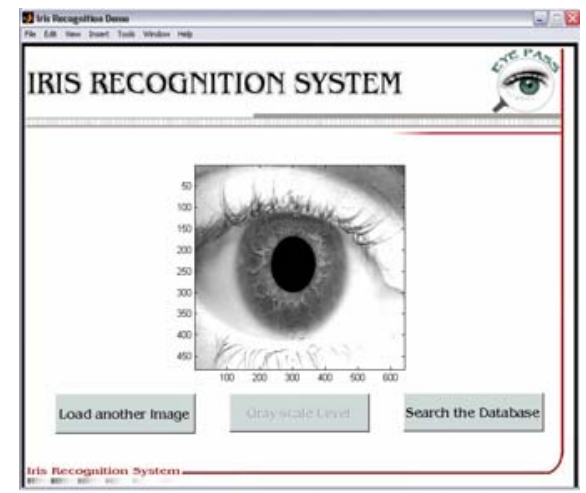

(a)

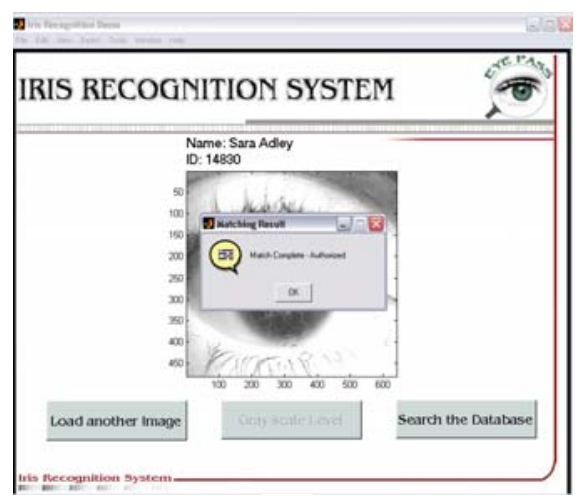

(b)

Fig. 13 Authorized individuals

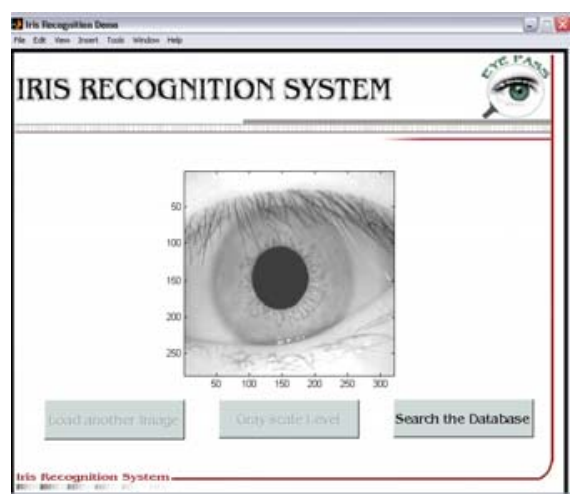

(a)

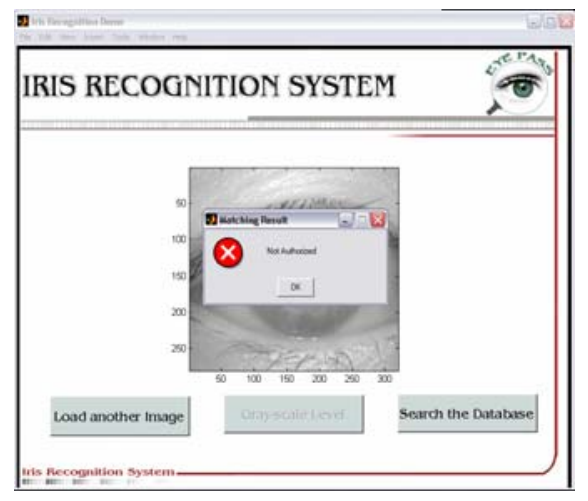

(b)

Fig. 14 Unauthorized individual

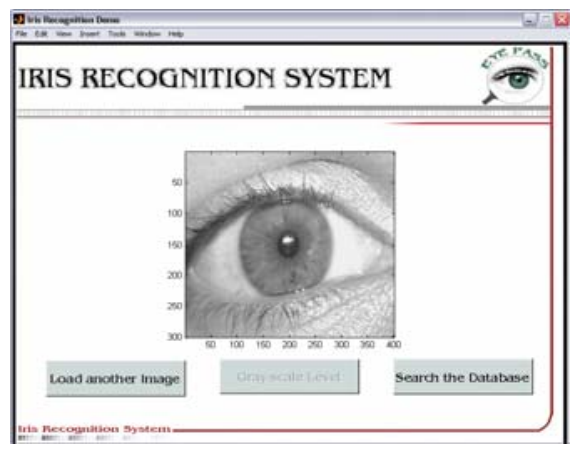

(a)

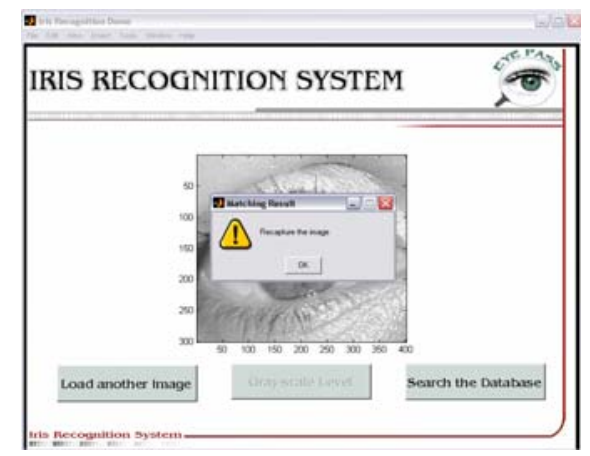

(b)

Fig. 15 Rejected image due to illumination affects: image must recaptured 


\section{CONCLUSIONS}

With the increasing emphasis on security, automated personal identification based on biometrics has been an active topic in pattern recognition. Recently, iris recognition has received increasing attention due to its high reliability. In this paper, an image enhancement algorithm was applied on the captured image before applying any process in order to get more accurate image feature results. In addition, we developed a new iris recognition method, which analyzes local variations to characterize the details of the iris. The method first constructs a set of 1-D Feature Selection Vector (FSV) that containing the majority of local variations of the iris, and then calculates Pearson's product-moment correlation coefficient between input FSV and FSV's in database. On the CASIA Iris Database of 2255 images from 213 different subjects, the proposed method achieves encouraging results.

\section{REFERENCES}

[1] Bae K., Noh S., and Kim J., "Iris feature extraction using independent component analysis", in Proc. $4^{\text {th }}$ Int. Conf. Audio-and Video-Based Biometric Person Authentication, pp. 838-844, (2003).

[2] Boles W. and Boashash B., "A human identification technique using images of the iris and wavelet transform", IEEE Trans. Signal Processing, Vol. 46, pp. 1185-1188, (1998).

[3] Castleman K. R., "Digital Image Processing", Prentice Hall, New Jersey, (1996).

[4] Daugman J., "Biometric personal identification system based on iris analysis" U.S. Patent 5291 560, (1994).

[5] Daugman J., "Demodulation by complex-valued wavelets for stochastic pattern recognition", Int. J. Wavelets, Multi-Res. and Info. Processing, vol. 1, no. 1, pp. 1-17, (2003).

[6] Daugman J., "High confidence visual recognition of persons by a test of statistical independence", IEEE Trans. Pattern Analy. Machine Intell., vol. 15, pp. 1148-1161, (1993).

[7] Daugman J., "Statistical richness of visual phase information: update on recognizing persons by iris patterns", Int. J. Comput. Vis., vol. 45, no. 1, pp. 25-38, (2001).

[8] Flom L. and Safir A., "Iris Recognition system", U.S. Patent 4641 394, (1987).

[9] Hugo Proença and Luís A. Alexandre, "UBIRIS: a noisy iris image database", in Lecture Notes in Computer Science - ICIAP 2005: 13th International Conference on Image Analysis and Processing, Cagliari, Italy, September 6-8, vol. 1, page 970-977, (2005).

[10] Jain A., Bolle R., and Pankanti S., Eds., Biometrics: Personal Identification in a Networked Society. Norwell, MA: Kluwer, (1999).

[11] Johnson R., "Can iris patterns be used to identify people?", Chemical and Laser Sciences Division LA-12 331-PR, Los Alamos Nat. Lab., Los Alamos, CA, (1991).

[12] Kong W., Zhang D., "Accurate iris segmentation based on novel reflection and eyelash detection model". Proceedings of 2001 International Symposium on Intelligent Multimedia, Video and Speech Processing, Hong Kong, (2001).

[13] Kumar B., Xie C., and Thornton J., "Iris verification using correlation filters," in Proc. 4th Int. Conf. Audio- and Video-Based Biometric Person Authentication, pp. 697-705, (2003).

[14] Ma L., Wang Y., and Tan T., "Efficient Iris Recognition by Characterizing Key Local Variations", IEEE Trans. on Image Processing, vol. 13, June, no. 6, pp. 739-750, (2004). 
[15] Ma L., Wang Y., and Tan T., "Iris recognition using circular symmetric filters", in Proc. 16th Int. Conf. Pattern Recognition, vol. II, pp. 414-417, (2002).

[16] Mansfield A. and Wayman J., "Best practice standards for testing and reporting on biometric device performance," Nat. Physical Lab., Middlesex, U.K., (2002).

[17] Mansfield T., Kelly G., Chandler D., and Kane J., "Biometric product testing final report", Nat. Physical Lab., Middlesex, U.K., (2001).

[18] Park C., Lee J., Smith M., and Park K., "Iris-based personal authentication using a normalized directional energy feature," in Proc. 4th Int. Conf. Audio- and Video-Based Biometric Person Authentication, pp. 224-232, (2003).

[19] Poursaberi A., Araabi B., "A Novel Iris Recognition System Using Morphological Edge Detector and Wavelet Phase Features", ICGST International Journal on Graphics, Vision and Image Processing, , Volume 6, pp. 9-15, (2005).

[20] Sanchez-Reillo R. and Sanchez-Avila C., "Iris recognition with low template size", in Proc. Int. Conf. Audio and Video-Based Biometric Person Authentication, pp. 324-329, (2001).

[21] Tisse C., Martin L., Torres L., and Robert M., "Person identification technique using human iris recognition" in Proc. Vision Interface, pp. 294-299, (2002).

[22] Wildes R., "Iris recognition: an emerging biometric technology", Proc. IEEE, vol. 85, pp. 1348-1363, (1997).

[23] Wildes R., Asmuth J., Hsu S., Kolczynski R., Matey J., and Mcbride S., “A machinevision system for iris recognition”, Mach. Vis. Applic., vol. 9, pp. 1-8, (1996).

[24] Wildes R., Asmuth J., Hsu S., Kolczynski R., Matey J., and Mcbride S., "Automated, noninvasive iris recognition system and method", U.S. Patent 5572 596, (1996).

[25] Zhang D., "Automated Biometrics: Technologies and Systems", Norwell, MA: Kluwer, (2000).

[26] Zhu Y., Tan T., and Wang Y., "Biometric personal identification based on iris patterns", in Proc. Int. Conf. Pattern Recognition, vol. II, pp. 805-808, (2000). 